\title{
Clinical Impact of Hemorheology Assessed Using the Microchannel Array Flow Analyzer on Renal Resistive Index in Patients With Type 2 Diabetes Mellitus
}

\author{
Takashi Hitsumoto
}

\begin{abstract}
Background: The renal resistive index (RRI) assessed using ultrasonography is reportedly associated with not only kidney function but also cardiovascular disease incidence. In contrast, hemorheology assessed using the microchannel array flow analyzer (MC-FAN) has demonstrated the significance of cardiovascular risk factors in recent clinical studies. This cross-sectional study aimed to clarify the clinical impact of hemorheology assessed using MC-FAN on RRI in patients with type 2 diabetes mellitus from the perspective of primary prevention of cardiovascular events.
\end{abstract}

Methods: In total, 302 outpatients undergoing treatment for type 2 diabetes mellitus (121 males and 181 females; mean age \pm standard deviation, $66 \pm 12$ years) with no history of cardiovascular diseases were enrolled. The whole blood passage time (WBPT) was assessed using MC-FAN as a marker of hemorheology, and the relationship between RRI and various clinical parameters including WBPT was examined.

Results: A significant positive correlation was observed between WBPT and RRI (correlation coefficient $=0.48 ; \mathrm{P}<0.001$ ). Furthermore, the multiple regression analysis demonstrated that WBPT was selected as an independent variable for RRI as a subordinate factor $(\beta=0.37 ; \mathrm{P}<0.001)$. Patients with high WBPT $(\geq 70 \mathrm{~s})$ and median WBPT (50.2 - 69.6 s) had a significantly higher risk (odds ratio (OR): 7.9; 95\% confidence interval (CI): $2.4-19.6$; $>0.01$ and OR: 2.1; 95\% CI: $1.1-7.8 ; \mathrm{P}<0.05$, respectively) of having a high RRI $(\geq$ $0.70)$ than those with low WBPT $(\leq 50.0 \mathrm{~s})$.

Conclusions: The results of this study indicate that hemorheology assessed using MC-FAN is an important determining factor for RRI in patients with type 2 diabetes mellitus. Furthermore, we suggest that an increase in RRI can be prevented by maintaining WBPT $\leq 50.0 \mathrm{~s}$. Further investigation of a large number of prospective studies, including intervention therapies, will be required to confirm the results of this study.

Manuscript submitted September 1, 2017, accepted September 12, 2017

Hitsumoto Medical Clinic, 2-7-7, Takezakicyou, Shimonoseki City, Yamaguchi 750-0025, Japan. Email: thitsu@jcom.home.ne.jp

doi: https://doi.org/10.14740/jem448w
Keywords: Hemorheology; Microchannel method; Renal resistive index; Albuminuria; Skin autofluorescence; Oxidative stress; Type 2 diabetes mellitus

\section{Introduction}

Complications of kidney dysfunction are widely known to be associated with the prognosis of type 2 diabetes mellitus. In general, the kidney function is evaluated using biomarkers, such as estimated glomerular filtration rate (eGFR) and urinary albumin concentration, which are also well established as prognosis factors for cardiovascular events in patients with type 2 diabetes mellitus [1]. In addition, the renal resistive index (RRI), which is assessed using ultrasonography, has been reported as a novel marker of kidney function and a predictor of cardiovascular events $[2,3]$.

The impairment of hemorheology is considered as a crucial factor in the incidence of cardiovascular events, as well as atherosclerosis $[4,5]$. In recent years, the microchannel array flow analyzer (MC-FAN), a commercial device that assesses hemorheology using microscopic images, has been introduced to clinical settings [6]. MC-FAN has a simple methodology and is superior to other methods regarding the accuracy of channel dimensions and high reproducibility. Furthermore, some clinical studies have reported a significant relationship between an increase in the whole blood passage time (WBPT), which is measured using MC-FAN, and cardiovascular risk factors or coronary artery disease [7-11].

To the best of our knowledge, there are no reports on the relationship between WBPT and RRI in patients with type 2 diabetes mellitus. Therefore, this study aimed to elucidate the clinical impact of hemorheology assessed using MC-FAN on RRI in patients with type 2 diabetes mellitus from the perspective of primary prevention of cardiovascular events.

\section{Methods}

\section{Patients}

This cross-sectional study was conducted at Hitsumoto Medi- 
cal Clinic (Shimonoseki City, Japan) from February 2015 to January 2017. The study population comprised 302 outpatients (121 males and 181 females; mean age \pm standard deviation, 66 \pm 12 years) undergoing treatment for type 2 diabetes mellitus whose blood rheology was examined using MC-FAN. No patient had a history of cardiovascular events, such as coronary artery disease, cerebrovascular disease, peripheral artery disease, or heart failure. All participants provided informed consent, and the study protocol conformed to the ethical guidelines of the Declaration of Helsinki. Furthermore, this study was approved by the Local Ethics Committee of Hitsumoto Medical Clinic.

\section{Assessment of hemorheology using MC-FAN}

Hemorheology was assessed by measuring WBPT using an MC-FAN HR300 rheometer (MC Healthcare Inc., Tokyo, Japan), as previously reported $[6,10]$. The microchannel passage time for $100 \mu \mathrm{L}$ of physiological saline as a control was initially measured, followed by that for $100 \mu \mathrm{L}$ of heparinized blood samples obtained from the study participants. WBPT of the participants was corrected for the passage time of physiological saline. The microchannel formation was $7 \mu \mathrm{m}$ wide, $30 \mu \mathrm{m}$ long and $4.5 \mu \mathrm{m}$ deep. The WBPT measurements were performed within 60 min of blood sampling. Both inter- and intra-assay coefficients of variation for WBPT were $8 \%$ and $5 \%$, respectively.

\section{Evaluation of RRI}

Renal arteries were ultrasonographically examined using a 3.0 $\mathrm{MHz}$ convex probe (HI VISION Avius; Hitachi Medical Corporation, Tokyo, Japan). RRI was measured in a supine position during suspended respiration at the end of inspiration, as previously reported [12]. Using pulsed wave Doppler, blood flow velocities were measured from segmental arteries located in the upper, middle, and lower thirds of the kidney for the RRI analysis. RRI was automatically calculated using the following equation: (peak systolic velocity - minimum diastolic velocity)/peak systolic velocity. The three values were averaged to obtain the mean RRI for each kidney, and the average RRI value between the right and left kidney was used for analysis.

\section{Evaluation of cardiovascular risk factors}

The degree of obesity was measured using the body mass index, which was calculated as weight in kilograms divided by height in meters squared. Current smoking was defined as smoking at least one cigarette per day during the previous 28 days. The right brachial blood pressure was measured twice using a mercury sphygmomanometer with participants in the sitting position. An average of two readings was used to determine the systolic and diastolic blood pressures. The pulse pressure was calculated by the difference between the systolic and diastolic blood pressures. In addition, eGFR was calculated using the adjusted Modification of Diet in Renal Disease
Table 1. Baseline Clinical Characteristics

\begin{tabular}{|c|c|}
\hline n (male/female) & $302(121 / 181)$ \\
\hline Age (years) & $66 \pm 12$ \\
\hline Body mass index & $23.6 \pm 3.8$ \\
\hline Current smoker, n (\%) & $57(19)$ \\
\hline Systolic blood pressure (mm Hg) & $143 \pm 9$ \\
\hline Diastolic blood pressure (mm Hg) & $90 \pm 12$ \\
\hline Pulse pressure $(\mathrm{mm} \mathrm{Hg})$ & $53 \pm 13$ \\
\hline Pulse rate (/min) & $68 \pm 11$ \\
\hline White blood cell $(/ \mu \mathrm{L})$ & $6,490 \pm 1,220$ \\
\hline Red blood cell $\left(10^{4} / \mu \mathrm{L}\right)$ & $420 \pm 40$ \\
\hline Hematocrit $(\%)$ & $38.0 \pm 4.0$ \\
\hline Platelet $\left(10^{4} / \mu \mathrm{L}\right)$ & $21.2 \pm 6.5$ \\
\hline Fasting blood glucose (mg/dL) & $134 \pm 24$ \\
\hline Immunoreactive insulin $(\mu \mathrm{g} / \mathrm{mL})$ & $7.3 \pm 4.4$ \\
\hline HOMA-IR & $2.5 \pm 1.5$ \\
\hline Hemoglobin A1c (\%) & $7.1 \pm 1.3$ \\
\hline Skin autofluorescence (AU) & $2.6 \pm 0.6$ \\
\hline Total cholesterol (mg/dL) & $213 \pm 40$ \\
\hline LDL-cholesterol (mg/dL) & $135 \pm 35$ \\
\hline Triglyceride (mg/dL) & $129 \pm 63$ \\
\hline HDL-cholesterol (mg/dL) & $52 \pm 15$ \\
\hline eGFR (mL/min/1.73 m²) & $63.5 \pm 25.7$ \\
\hline Log-urinary albumin (mg/g Cr) & $1.52 \pm 0.53$ \\
\hline d-ROMs test (U Carr) & $334 \pm 99$ \\
\hline WBPT (s) & $59.3 \pm 16.1$ \\
\hline RRI & $0.70 \pm 0.08$ \\
\hline \multicolumn{2}{|l|}{ Medication } \\
\hline Sulfonylurea, n (\%) & $157(52)$ \\
\hline Metformin, n (\%) & $81(27)$ \\
\hline DPP-4 inhibitor, n (\%) & $172(57)$ \\
\hline Insulin, $\mathrm{n}(\%)$ & $21(7)$ \\
\hline RAS inhibitor, n (\%) & $166(55)$ \\
\hline Statin, n (\%) & $163(54)$ \\
\hline
\end{tabular}

Continuous values are mean \pm SD. HOMA-IR: homeostasis assessment insulin resistance; LDL: low-density lipoprotein; HDL: high-density lipoprotein; eGFR: estimated glomerular filtration rate; d-ROMs: derivatives of reactive oxygen metabolites; RRI: renal resistive index; WBPT: whole blood passage time; RAS: renin-angiotensin system; DPP: dipeptidyl peptidase.

Study equation, which was proposed by the working group of the Japanese Chronic Kidney Disease Initiative [13]. Urinary albumin excretion was evaluated using a commercial kit (Siemens/Bayer DCA 2000+ Analyzer; Siemens Healthineers, Tokyo, Japan). Skin autofluorescence (AF), which reflects the accumulation of advanced glycation end products (AGEs), was measured on the volar side of the forearm us- 


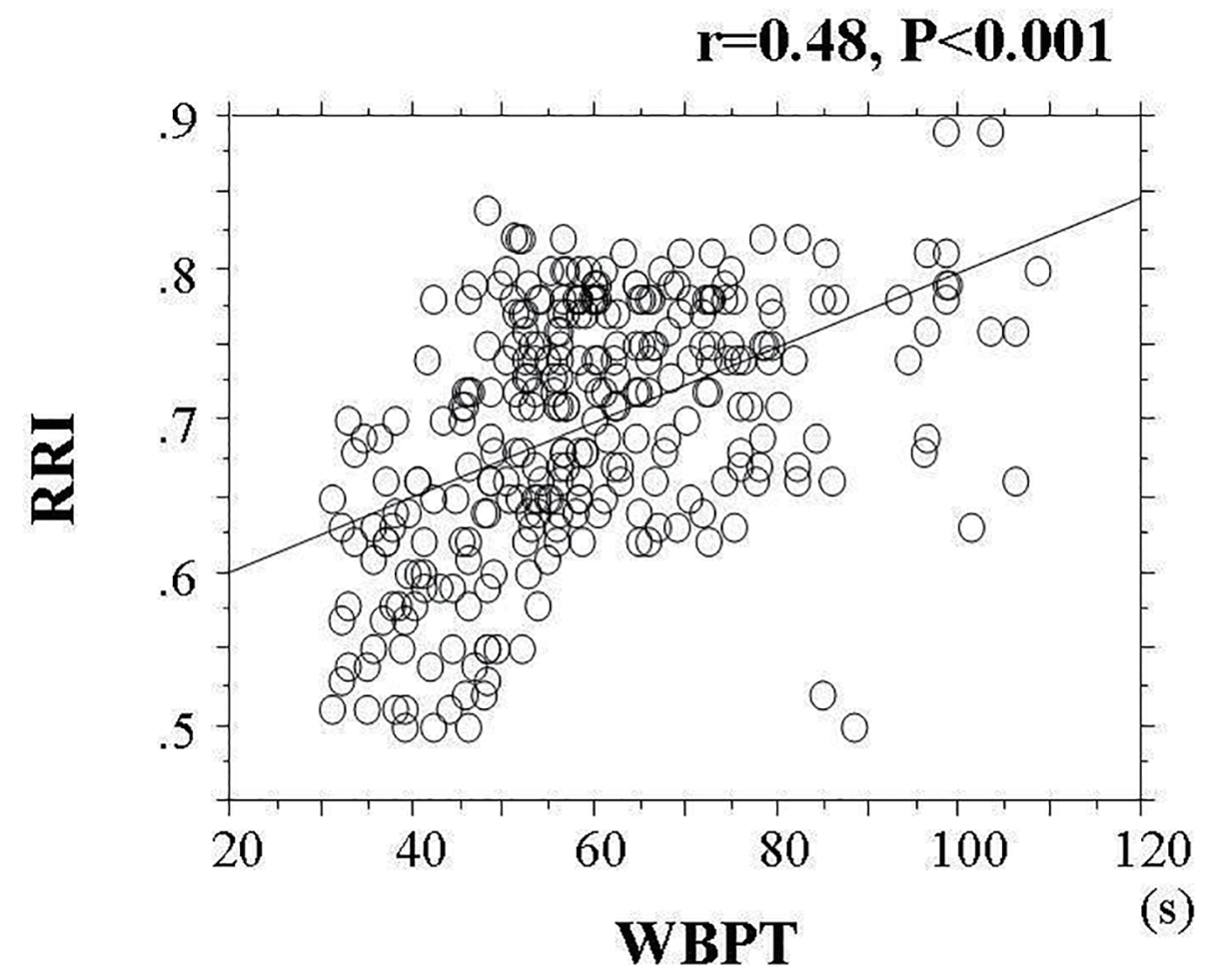

Figure 1. Correlation Between WBPT and RRI. The statistically significant positive correlation between WBPT and RRI $(r=0.48$, $P<0.001)$. WBPT: whole blood passage time; RRI: renal resistive index.

ing a commercial instrument (AGE Reader ${ }^{\mathrm{TM}}$; DiagnOptics, Groningen, the Netherlands), as previously described [14]. The blood cell count, plasma glucose concentration, plasma insulin concentration, hemoglobin A1c (HbA1c), serum lipid concentration, and derivatives of reactive oxygen metabolites (d-ROMs) were assessed using d-ROMS test. Blood samples were collected from antecubital veins in the morning after $12 \mathrm{~h}$ of fasting. Glucose and insulin concentrations were measured using the glucose oxidase method and an enzyme immunoassay, respectively. To assess insulin resistance, the homeostasis model assessment (HOMA-IR) was calculated as follows [15]: HOMA-IR $=$ (fasting glucose concentration $(\mathrm{mg} / \mathrm{dL}) \times$ fasting insulin concentration $(\mu \mathrm{g} / \mathrm{mL})) / 405$. HbAlc levels were expressed using the National Glycohemoglobin Standardization Program. Total cholesterol and triglyceride concentrations were evaluated using standard enzymatic methods. In addition, high- and low-density lipoprotein cholesterol concentrations were assessed using selective inhibition and the Friedewald formula, respectively [16]. Notably, participants with a serum triglyceride concentration of $\geq 400 \mathrm{mg} / \mathrm{dL}$ were excluded from the study because this method is accurate only below this concentration. The d-ROMs test, which assesses hydroperoxide levels, was used to measure the oxidative stress in vivo using a commercial device (Diacron, Grosseto, Italy) [17].

\section{Statistical analysis}

A commercially available statistical software program (Stat-
View-J 5.0; Hulinks Inc., Tokyo, Japan) was used for all statistical analyses. Continuous variables were expressed as mean $\pm \mathrm{SD}$. A simple regression analysis was performed using the Spearman's rank correlation, and the multivariate analysis was conducted using multiple regression analysis or multiple logistic regression analysis. $\mathrm{P}<0.05$ was considered statistically significant.

\section{Results}

Table 1 shows the baseline clinical characteristics. The mean and median values of WBPT were $59.3 \mathrm{~s}( \pm 16.1 \mathrm{~s}$; range, 31.1 - $108.6 \mathrm{~s})$ and $56.9 \mathrm{~s}$, respectively. The mean and median values of RRI were 0.70 ( \pm 0.08 ; range, $0.50-0.89)$ and 0.71 , respectively. Figure 1 shows the statistically significant positive correlation between WBPT and RRI. Table 2 displays correlations between RRI, WBPT, and various clinical parameters. RRI demonstrated a significant positive correlation with age, current smoking habits, pulse pressure, HbAlc, skin AF, urinary albumin concentration, and d-ROMs test. Conversely, RRI exhibited a significant negative correlation with diastolic blood pressure and eGFR. WBPT showed a significant positive correlation with body mass index, current smoking habits, hematocrit, $\mathrm{HbAlc}$, skin AF, serum triglyceride concentration, urinary albumin concentration, and d-ROMs test. Table 3 shows results of multiple regression analysis. The selection of seven explanatory factors was determined by either checking multicollinearity among variables or conducting stepwise 
Table 2. Relationship Between RRI, WBPT and Various Clinical Parameters

\begin{tabular}{|c|c|c|}
\hline & \multicolumn{2}{|c|}{$\mathbf{r}$} \\
\hline & RRI & WBPT \\
\hline Sex $($ Female $=0$, Male $=1)$ & -0.06 & 0.07 \\
\hline Age & $0.26 *$ & 0.06 \\
\hline Body mass index & 0.09 & $0.16^{* *}$ \\
\hline Current smoker $(\mathrm{No}=0, \mathrm{Yes}=1)$ & $0.12 * * *$ & $0.13 * * *$ \\
\hline Systolic blood pressure & 0.03 & 0.04 \\
\hline Diastolic blood pressure & $-0.28 *$ & 0.05 \\
\hline Pulse pressure & $0.36^{*}$ & 0.04 \\
\hline Pulse rate & 0.07 & 0.05 \\
\hline White blood cell & 0.06 & 0.06 \\
\hline Red blood cell & 0.10 & 0.10 \\
\hline Hematocrit & 0.11 & $0.13 * * *$ \\
\hline Platelet & 0.07 & 0.07 \\
\hline Fasting blood glucose & 0.11 & 0.10 \\
\hline Immunoreactive insulin & 0.05 & 0.05 \\
\hline HOMA-IR & 0.06 & 0.08 \\
\hline Hemoglobin A1c & $0.16^{* *}$ & $0.12 * * *$ \\
\hline Skin autofluorescence & $0.41 *$ & $0.41 *$ \\
\hline Total cholesterol & 0.03 & 0.02 \\
\hline LDL-cholesterol & 0.02 & 0.03 \\
\hline Triglyceride & 0.09 & $0.13 * * *$ \\
\hline HDL-cholesterol & -0.08 & 0.08 \\
\hline eGFR & $-0.20^{*}$ & -0.08 \\
\hline Log-urinary albumin & $0.34 *$ & $0.45 *$ \\
\hline d-ROMs test & $0.40 *$ & $0.41 *$ \\
\hline Sulfonylurea $($ no $=0$, yes $=1)$ & 0.03 & -0.08 \\
\hline Metformin $($ no $=0$, yes $=1)$ & -0.05 & -0.06 \\
\hline DPP-4 inhibitor $($ no $=0$, yes $=1)$ & -0.04 & -0.07 \\
\hline Insulin $($ no $=0$, yes $=1)$ & 0.08 & 0.09 \\
\hline RAS inhibitor $($ no $=0$, yes $=1)$ & -0.08 & -0.07 \\
\hline Statin $($ no $=0$, yes $=1)$ & -0.08 & -0.09 \\
\hline
\end{tabular}

$r$ expressed correlation coefficient. HOMA-IR: homeostasis assessment insulin resistance; LDL: low-density lipoprotein; HDL: high-density lipoprotein; d-ROMs: derivatives of reactive oxygen metabolites; eGFR: estimated glomerular filtration rate; DPP: dipeptidyl peptidase RAS: renin-angiotensin system. ${ }^{*} \mathrm{P}<0.001,{ }^{* *} \mathrm{P}<0.01,{ }^{* *} \mathrm{P}<0.05$.

method. The coefficient of determination $\left(\mathrm{R}^{2}\right)$ of this analysis was 0.33 , indicating that $33 \%$ of RRI as a subordinate factor was explained by the seven explanatory factors. In this analysis, WBPT, pulse pressure, urinary albumin concentration, skin $\mathrm{AF}, \mathrm{d}-\mathrm{ROMs}$ test, and age were selected as independent variables for RRI as a subordinate factor. To illustrate the simple threshold of WBPT for detecting high RRI (RRI $\geq 0.70$ ), participants were divided into three groups based on WBPT, and the multiple logistic regression analysis was performed (Fig.
Table 3. Multiple Regression Analysis for RRI

\begin{tabular}{lll} 
Explanatory factor & $\boldsymbol{\beta}$ & P value \\
\hline WBPT & 0.37 & $<0.001$ \\
Pulse pressure & 0.24 & $<0.001$ \\
Log-urinary albumin & 0.22 & $<0.001$ \\
Skin autofluorescence & 0.21 & $<0.001$ \\
d-ROMs test & 0.15 & $<0.01$ \\
Age & 0.12 & $<0.05$ \\
eGFR & -0.09 & 0.061 \\
\hline
\end{tabular}

$R^{2}=0.33$. RRI: renal resistive index; WBPT: whole blood passage time; d-ROMs: derivatives of reactive oxygen metabolites; eGFR: estimated glomerular filtration rate; $\beta$ : standardized regression coefficient; $R^{2}$ : coefficient of determination.

2). Patients with high WBPT ( $\geq 70 \mathrm{~s})$ and median WBPT (50.2 - $69.6 \mathrm{~s}$ ) had a significantly higher risk (odds ratio (OR): 7.9; 95\% confidence interval (CI): 2.4 - 19.6, $\mathrm{P}<0.01$ and OR: 2.1; 95\% CI: $1.1-7.8 ; \mathrm{P}<0.05$, respectively) of having a high RRI than patients with low WBPT $(\leq 50.0 \mathrm{~s})$.

\section{Discussion}

Previous studies have reported that the RRI value is mainly affected by age and pulse pressure [18, 19]. In this study, the multivariate analysis also indicated that age and pulse pressure were independent variables for RRI. Furthermore, the novelty of this study was elucidating the significant relationships between hemorheology assessed by microchannel methods and RRI. In addition, albuminuria, skin AGEs levels, and oxidative stress were selected as significant factors for RRI in this study.

RRI is considered to reflect renovascular resistance distal from the point of examination. Ikee et al reported the relationship between RRI and renal histopathological data, including glomerular sclerosis, interstitial fibrosis/tubular atrophy, interstitial infiltration, and arteriosclerosis. They concluded that the multivariate analysis selected only arteriosclerosis as an independent variable for RRI [20]. In addition, this study indicated that WBPT had the highest $\beta$-value for RRI in the multiple regression analysis. Linde et al reported a significant relationship between blood viscosity and peripheral vascular resistance in vivo [21]. Thus, the results of this and previous studies indicated that an increase in RRI is caused by both arteriosclerosis and the impairment of blood rheology of renal arteries.

In this study, both RRI and WBPT had significant positive correlations with the urinary albumin concentration. Although the mechanisms underlying the development of albuminuria are not completely understood, recent basic studies using animal models have reported that the impairment of preglomerular small vessels, particularly the perforating arteries, plays a crucial role in the incidence of urinary albumin [22]. Conversely, hemorheology assessed using MC-FAN is an in vitro measurement that uses artificial blood vessels, with a $7 \mu \mathrm{m}$ wide, $30 \mu \mathrm{m}$ long, and $4.5 \mu \mathrm{m}$ deep vessel lumen. Thus, assessing hemorheology using MC-FAN is assumed to correspond 


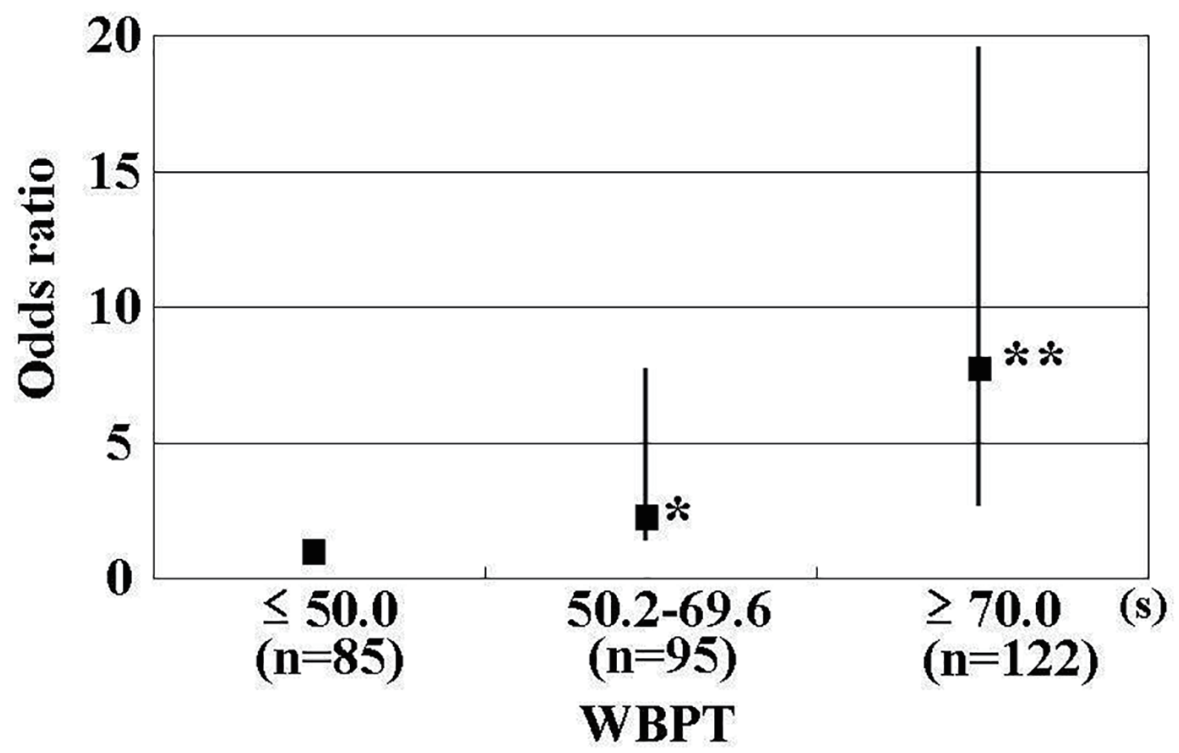

Figure 2. Results of Multiple Logistic Regression Analysis for High RRI. High RRI was defined as an RRI $\geq 0.70$. Adjustment factors were age, pulse pressure, skin autofluorescence, urinary albumin excretion, and d-ROMs. ${ }^{*} P<0.01 \mathrm{vs} . \leq 50.0 \mathrm{~s},{ }^{* \star} \mathrm{P}<0.001$ vs. $\leq 50.0$ s. RRI: renal resistive index; WBPT: whole blood passage time; d-ROMs: derivatives of reactive oxygen metabolites.

to small vessels, including perforating arteries. Therefore, the impairment of blood rheology in a small-sized vessel may be affected by the production of albuminuria in addition to an increase in RRI. It is well known that endothelial dysfunction plays a major role in the occurrence of cardiovascular events, and some studies have indicated that the urinary albumin concentration is closely associated with systemic endothelial dysfunction [23]. Yagi et al reported a significant association between increased WBPT and endothelial dysfunction using flow-mediated dilatation of the brachial artery [24]. Therefore, a significant relationship observed between RRI and urinary albumin excretion in this study may be partly attributable to the systemic endothelial dysfunction due to the impairment of hemorheology.

Among the factors of glucose-related parameters, only skin AF was selected as an independent variable for RRI. Furthermore, skin AF had a significant positive correlation with WBPT. It is well known that AGEs play a crucial role in the pathogenesis of kidney disease $[25,26]$. In contrast, the skin pentosidine level, which is a major component of AGEs on the volar side of the forearm, has been found to correlate with skin AF in skin biopsy [27]. Small-sized vessels are considered to be present in a portion of skin biopsies along with systemic vessels such as the kidney, heart, and brain. In addition, basic studies have reported that AGEs influence hemorheology by mechanisms such as leukocyte-endothelial interaction and platelet aggregation [28, 29]. Thus, the results of this study and that of previous studies may indicate that AGEs and the impairment of hemorheology associated with each other in systemic small-sized vessels consequently lead to an increased systemic vascular resistance, including the kidneys, in patients with type 2 diabetes mellitus.

A number of studies have reported an association between oxidative stress and the pathogenesis of diabetes mellitus [30,
31]. In this study, the d-ROMs test, as a marker of the oxidative stress in vivo, also exhibited a significant correlation with RRI by the multivariate analysis. Thus, oxidative stress is considered as one of the important determining factors for an increase in RRI in type 2 diabetes mellitus. Conversely, the d-ROMs test also demonstrated a significant correlation with WBPT. Some basic and clinical studies have reported that oxidative stress plays an important role in blood rheology and causes the impairment of hemorheology through several mechanisms, such as platelet aggregation and increased plasma viscosity [8, $11,32-35]$. Furthermore, the results of this study indicate that oxidative stress may cause an increase in RRI by impairing blood rheology in small renal arteries.

Evidently, the simple cut-off levels of WBPT values for predicting the high RRI in clinical settings are useful. This study clarifies the clinical usefulness of assessing WBPT to detect RRI as high as $\geq 0.70$, which is shown to be discriminatory of an increased RRI level $[2,36]$. Participants were divided into three groups based on simple WBPT values, and the multiple logistic regression analysis was performed. The results of this study indicate that patients with WBPT $\geq 70.0 \mathrm{~s}$ or 50.2 - $69.6 \mathrm{~s}$ exhibited a significantly higher risk of high RRI than patients with WBPT $\leq 50.0 \mathrm{~s}$. Although this was a crosssectional study, we believe that it is possible to evaluate the risk of progression of high RRI in patients with type 2 diabetes mellitus by measuring WBPT. Some clinical studies have indicated that medications, such as antidiabetic drugs, antihypertensive drugs, and antihyperlipidemic drugs, and smoking cessation, improve hemorheology, even though no statistically significant associations between these factors and WBPT were observed in this cross-sectional study [37-40]. We suggest that an increase in RRI can be prevented by interventions such as medication or lifestyle modification in patients with type 2 diabetes mellitus to maintain WBPT $\leq 50.0 \mathrm{~s}$. 
This study has several limitations. First, the drug treatments for diabetes mellitus, hypertension, and dyslipidemia might have influenced the study results. Second, angiography, computed tomography, magnetic resonance imaging, and echocardiography were not performed. Therefore, asymptomatic cardiovascular diseases might have gone undetected. Third, hemorheology assessed using MC-FAN is an in vitro assessment that uses artificial blood vessels; therefore, the obtained hemorheological data might differ from those obtained in vivo because of the influence of vascular factors, such as endothelial cells or smooth muscle cells. However, the results of this study indicate that WBPT is an important marker that is associated with an increase in RRI or risk factor of cardiovascular disease incidence. Finally, this study was cross-sectional and conducted at a single unit with a small sample volume. An examination of a large number of prospective studies, including intervention therapies, will be required to confirm the results of this study.

\section{Conclusions}

In conclusion, the results of this study indicate that hemorheology assessed using MC-FAN is one of the important determining factors for RRI in patients with type 2 diabetes mellitus. Furthermore, we suggest that an increase in RRI can be prevented by maintaining WBPT $\leq 50.0 \mathrm{~s}$. Further investigation of a large number of prospective studies, including intervention therapies, will be required to confirm the results of this study.

\section{Competing Interests}

Author has no competing interests.

\section{Grant Support}

None.

\section{Financial Disclosure}

The author has reported no financial support.

\section{References}

1. Bouchi R, Babazono T, Yoshida N, Nyumura I, Toya K, Hayashi T, Hanai K, et al. Association of albuminuria and reduced estimated glomerular filtration rate with incident stroke and coronary artery disease in patients with type 2 diabetes. Hypertens Res. 2010;33(12):1298-1304.

2. Ozmen ND, Mousa U, Aydin Y, Deren T, Unlu EB. Association of the renal resistive index with microvascular complications in type 2 diabetic subjects. Exp Clin Endocrinol Diabetes. 2015;123(2):112-117.

3. Viazzi F, Leoncini G, Derchi LE, Pontremoli R. Ultra- sound Doppler renal resistive index: a useful tool for the management of the hypertensive patient. J Hypertens. 2014;32(1):149-153.

4. Cowan AQ, Cho DJ, Rosenson RS. Importance of blood rheology in the pathophysiology of atherothrombosis. Cardiovasc Drugs Ther. 2012;26(4):339-348.

5. Tzoulaki I, Murray GD, Lee AJ, Rumley A, Lowe GD, Fowkes FG. Relative value of inflammatory, hemostatic, and rheological factors for incident myocardial infarction and stroke: the Edinburgh Artery Study. Circulation. 2007;115(16):2119-2127.

6. Kikuchi Y, Sato K, Mizuguchi Y. Modified cell-flow microchannels in a single-crystal silicon substrate and flow behavior of blood cells. Microvasc Res. 1994;47(1):126139.

7. Satoh N, Kotani K, Wada H, Himeno A, Shimada S, Sasaki Y, Yamada K, et al. Unfavorable blood rheology is closely associated with arterial stiffness in obese patients. Endocr J. 2009;56(7):915-918.

8. Hitsumoto T. Factors affecting impairment of blood rheology in obese subjects. J Cardiol. 2012;60(5):401-406.

9. Matsuo K, Ueda Y, Nishio M, Hirata A, Asai M, Nemoto $\mathrm{T}$, Kashiwase K, et al. Thrombogenic potential of whole blood is higher in patients with acute coronary syndrome than in patients with stable coronary diseases. Thromb Res. 2011;128(3):268-273.

10. Freestone T, Turner RJ, Higman DJ, Lever MJ, Powell JT. Influence of hypercholesterolemia and adventitial inflammation on the development of aortic aneurysm in rabbits. Arterioscler Thromb Vasc Biol. 1997;17(1):10-17.

11. Hitsumoto T. Relationship between cardiovascular risk factors and hemorheology assessed by microchannel method in patients with type 2 diabetes mellitus. Diabetology International. 2017;8(3):316-322.

12. Tublin ME, Bude RO, Platt JF. Review. The resistive index in renal Doppler sonography: where do we stand? AJR Am J Roentgenol. 2003;180(4):885-892.

13. Imai E, Horio M, Nitta K, Yamagata K, Iseki K, Hara $\mathrm{S}$, Ura $\mathrm{N}$, et al. Estimation of glomerular filtration rate by the MDRD study equation modified for Japanese patients with chronic kidney disease. Clin Exp Nephrol. 2007;11(1):41-50.

14. Meerwaldt R, Links TP, Graaff R, Hoogenberg K, Lefrandt JD, Baynes JW, Gans RO, et al. Increased accumulation of skin advanced glycation end-products precedes and correlates with clinical manifestation of diabetic neuropathy. Diabetologia. 2005;48(8):1637-1644.

15. Matthews DR, Hosker JP, Rudenski AS, Naylor BA, Treacher DF, Turner RC. Homeostasis model assessment: insulin resistance and beta-cell function from fasting plasma glucose and insulin concentrations in man. Diabetologia. 1985;28(7):412-419.

16. Friedewald WT, Levy RI, Fredrickson DS. Estimation of the concentration of low-density lipoprotein cholesterol in plasma, without use of the preparative ultracentrifuge. Clin Chem. 1972;18(6):499-502.

17. Cesarone MR, Belcaro G, Carratelli M, Cornelli U, De Sanctis MT, Incandela L, Barsotti A, et al. A simple test to monitor oxidative stress. Int Angiol. 1999;18(2):127-130. 
18. Heine GH, Reichart B, Ulrich C, Kohler H, Girndt M. Do ultrasound renal resistance indices reflect systemic rather than renal vascular damage in chronic kidney disease? Nephrol Dial Transplant. 2007;22(1):163-170.

19. Lubas A, Kade G, Niemczyk S. Renal resistive index as a marker of vascular damage in cardiovascular diseases. Int Urol Nephrol. 2014;46(2):395-402.

20. Ikee R, Kobayashi S, Hemmi N, Imakiire T, Kikuchi Y, Moriya H, Suzuki S, et al. Correlation between the resistive index by Doppler ultrasound and kidney function and histology. Am J Kidney Dis. 2005;46(4):603-609.

21. Linde T, Sandhagen B, Hagg A, Morlin C, Wikstrom B, Danielson BG. Blood viscosity and peripheral vascular resistance in patients with untreated essential hypertension. J Hypertens. 1993;11(7):731-736.

22. Ito S, Nagasawa T, Abe M, Mori T. Strain vessel hypothesis: a viewpoint for linkage of albuminuria and cerebrocardiovascular risk. Hypertens Res. 2009;32(2):115-121.

23. Pedrinelli R, Giampietro O, Carmassi F, Melillo E, Dell'Omo G, Catapano G, Matteucci E, et al. Microalbuminuria and endothelial dysfunction in essential hypertension. Lancet. 1994;344(8914):14-18.

24. Yagi H, Sumino H, Aoki T, Tsunekawa K, Araki O, Kimura T, Nara M, et al. Impaired blood rheology is associated with endothelial dysfunction in patients with coronary risk factors. Clin Hemorheol Microcirc. 2016;62(2):139150.

25. Bohlender JM, Franke S, Stein G, Wolf G. Advanced glycation end products and the kidney. Am J Physiol Renal Physiol. 2005;289(4):F645-659.

26. Thomas MC. Advanced glycation end products. Contrib Nephrol. 2011;170:66-74.

27. Meerwaldt R, Graaff R, Oomen PHN, Links TP, Jager JJ, Alderson NL, Thorpe SR, et al. Simple non-invasive assessment of advanced glycation endproduct accumulation. Diabetologia. 2004;47(7):1324-1330.

28. Morigi M, Angioletti S, Imberti B, Donadelli R, Micheletti G, Figliuzzi M, Remuzzi A, et al. Leukocyte-endothelial interaction is augmented by high glucose concentrations and hyperglycemia in a NF-kB-dependent fashion. J Clin Invest. 1998;101(9):1905-1915.

29. Hasegawa Y, Suehiro A, Higasa S, Namba M, Kakishita E. Enhancing effect of advanced glycation end products on serotonin-induced platelet aggregation in patients with diabetes mellitus. Thromb Res. 2002;107(6):319-323.

30. Zephy D, Ahmad J. Type 2 diabetes mellitus: Role of melatonin and oxidative stress. Diabetes Metab Syndr. 2015;9(2):127-131.

31. Gupta S, Gambhir JK, Kalra O, Gautam A, Shukla K, Mehndiratta M, Agarwal S, et al. Association of biomarkers of inflammation and oxidative stress with the risk of chronic kidney disease in Type 2 diabetes mellitus in North Indian population. J Diabetes Complications. 2013;27(6):548-552.

32. Richards RS, Nwose EU. Blood viscosity at different stages of diabetes pathogenesis. Br J Biomed Sci. 2010;67(2):67-70.

33. Gyawali P, Richards RS. Association of altered hemorheology with oxidative stress and inflammation in metabolic syndrome. Redox Rep. 2015;20(3):139-144.

34. Tzoulaki I, Murray GD, Lee AJ, Rumley A, Lowe GD, Fowkes FG. Inflammatory, haemostatic, and rheological markers for incident peripheral arterial disease: Edinburgh Artery Study. Eur Heart J. 2007;28(3):354-362.

35. Podrez EA, Byzova TV, Febbraio M, Salomon RG, Ma Y, Valiyaveettil M, Poliakov E, et al. Platelet CD36 links hyperlipidemia, oxidant stress and a prothrombotic phenotype. Nat Med. 2007;13(9):1086-1095.

36. Afsar B, Elsurer R, Sezer S, Ozdemir FN. Insulin resistance is associated with increased renal resistive index independent of other factors in newly diagnosed type 2 diabetes mellitus and hypertensive patients. Metabolism. 2010;59(2):279-284.

37. Berndt-Zipfel C, Michelson G, Dworak M, Mitry M, Loffler A, Pfutzner A, Forst T. Vildagliptin in addition to metformin improves retinal blood flow and erythrocyte deformability in patients with type 2 diabetes mellitus results from an exploratory study. Cardiovasc Diabetol. 2013;12:59.

38. Yamada K, Hirayama T, Hasegawa Y. Antiplatelet effect of losartan and telmisartan in patients with ischemic stroke. J Stroke Cerebrovasc Dis. 2007;16(5):225-231.

39. van der Loo B, Spring S, Koppensteiner R. High-dose atorvastatin treatment in patients with peripheral arterial disease: effects on platelet aggregation, blood rheology and plasma homocysteine. Clin Hemorheol Microcirc. 2011;47(4):241-251.

40. Shimada S, Hasegawa K, Wada H, Terashima S, SatohAsahara N, Yamakage H, Kitaoka S, et al. High blood viscosity is closely associated with cigarette smoking and markedly reduced by smoking cessation. Circ J. 2011;75(1):185-189. 